\title{
Utilizing the Updated Gamma-Ray Bursts and Type Ia Supernovae to Constrain the Cardassian Expansion Model and Dark Energy
}

\author{
Jun-Jie Wei, ${ }^{1,2}$ Qing-Bo $\mathrm{Ma}^{1,2}$ and Xue-Feng $\mathrm{Wu}^{1,3,4}$ \\ ${ }^{1}$ Purple Mountain Observatory, Chinese Academy of Sciences, Nanjing 210008, China \\ ${ }^{2}$ University of Chinese Academy of Sciences, Beijing 100049, China \\ ${ }^{3}$ Chinese Center for Antarctic Astronomy, Nanjing 210008, China \\ ${ }_{4}^{4}$ Joint Center for Particle, Nuclear Physics and Cosmology, Nanjing University-Purple Mountain Observatory, \\ Nanjing 210008, China
}

Correspondence should be addressed to Xue-Feng Wu; xfwu@pmo.ac.cn

Received 1 December 2014; Accepted 2 April 2015

Academic Editor: Gary Wegner

Copyright (c) 2015 Jun-Jie Wei et al. This is an open access article distributed under the Creative Commons Attribution License, which permits unrestricted use, distribution, and reproduction in any medium, provided the original work is properly cited.

We update gamma-ray burst (GRB) luminosity relations among certain spectral and light-curve features with 139 GRBs. The distance modulus of $82 \mathrm{GRBs}$ at $z>1.4$ can be calibrated with the sample at $z \leq 1.4$ by using the cubic spline interpolation method from the Union2.1 Type Ia supernovae (SNe Ia) set. We investigate the joint constraints on the Cardassian expansion model and dark energy with 580 Union2.1 SNe Ia sample $(z<1.4)$ and 82 calibrated GRBs' data $(1.4<z \leq 8.2)$. In $\Lambda$ CDM, we find that adding 82 high- $z$ GRBs to 580 SNe Ia significantly improves the constraint on $\Omega_{m}-\Omega_{\Lambda}$ plane. In the Cardassian expansion model, the best fit is $\Omega_{m}=0.24_{-0.15}^{+0.15}$ and $n=0.16_{-0.52}^{+0.30}(1 \sigma)$, which is consistent with the $\Lambda$ CDM cosmology $(n=0)$ in the $1 \sigma$ confidence region. We also discuss two dark energy models in which the equation of state $w(z)$ is parameterized as $w(z)=w_{0}$ and $w(z)=w_{0}+w_{1} z /(1+z)$, respectively. Based on our analysis, we see that our universe at higher redshift up to $z=8.2$ is consistent with the concordance model within $1 \sigma$ confidence level.

\section{Introduction}

In recent years, the combined observations of nearby and distant Type Ia supernovae (SNe Ia) have provided strong evidence for the current accelerated expansion of the universe [1-3]. The cause of the acceleration remains unknown. Many authors suggest that the composition of the universe may consist of an extra component called dark energy, which may explain the acceleration of the universe at the current epoch. For example, the dark energy model with a constant equation of state $P / \rho \equiv w=-1$ is one of the several possible explanations for the acceleration, while other models suggest that dark energy changes with time, and there are many ways to characterize the time variation of dark energy. Here, we adopt a simple model in which the dark energy equation of state can be parameterized by $P / \rho \equiv w(z)=w_{0}+w_{1} z /(1+$ $z)=w_{0}+w_{1}(1-a)[4,5]$, where $w_{0}$ is constant, $w_{1}$ represents the time dependence of dark energy, and $a=1 /(1+z)$ is the scale factor. In addition, models where general relativity is modified can also drive universe acceleration, such as the Cardassian expansion model which is a possible alternative for explaining the acceleration of the universe that invokes no vacuum energy [6].

SNe Ia have been considered a perfect standard candle to measure the geometry and dynamics of the universe. Unfortunately, the farthest SNe Ia detected so far is only at $z=1.914$ [7]. It is difficult to observe $\mathrm{SNe}$ at $z>2$, even with excellent space-based platforms such as SNAP [8]. And this is quite limiting because much of the most interesting evolution of the universe occurred well before this epoch. Gamma-ray bursts (GRBs) are the most luminous transient events at cosmological distances. Owing to their high luminosities, GRBs can be detected out to very high redshifts [9]. In fact, the farthest burst detected so far is GRB 090423, which is at $z=8.2$ [10] 
(a photometric redshift of 9.4 for GRB 090429B was reported by [11]). Moreover, in contrast to SNe Ia, gamma-ray photons from GRBs are almost immune to dust extinction, so the observed gamma-ray flux is a direct measurement of the prompt emission energy. Hence, GRBs are potentially more promising standard candles than $\mathrm{SNe}$ Ia at higher redshifts. The possible use of GRBs as cosmological probes started to become reality after some empirical luminosity relations were discovered. These GRB luminosity relations have been proposed as distance indicators, such as the correlations $\tau_{\text {lag }}-$ $L$ [12], $V-L[13], E_{p}-E_{\text {iso }}[14], E_{p}-L[15,16], E_{p}-E_{\gamma}$ [17], and $\tau_{\mathrm{RT}}-L$ [18]. Here the time lag $\left(\tau_{\text {lag }}\right)$ is the time shift between the hard and soft light curves; the luminosity $(L)$ is the isotropic peak luminosity of a GRB; the variability $(V)$ of a burst denotes whether its light curve is spiky or smooth, and $V$ can be obtained by calculating the normalized variance of an observed light curve around a smoothed version of that light curve [13]; $\left(E_{p}\right)$ is the burst frame peak energy in the GRB spectrum; $\left(E_{\text {iso }}\right)$ is the isotropic equivalent gamma-ray energy; $\left(E_{\gamma}\right)$ is the collimation-corrected gamma-ray energy; and the minimum rise time $\left(\tau_{\mathrm{RT}}\right)$ in the gamma-ray light curve is the shortest time over which the light curve rises by half of the peak flux of the pulse. However, [19] found that the updated $V-L$ correlation was quite scattered. Its intrinsic scatter has been larger than the one that could be expected of a linear relation.

Generally speaking, with these luminosity indicators, one can make use of them as standard candles for cosmological research. For example, [20] constructed the first GRB Hubble diagram based on nine GRBs using two GRB luminosity indicators. With the $E_{p}-E_{\gamma}$ relation, [21] placed tight constraints on cosmological parameters and dark energy. Reference [22] used a model-independent multivariable GRB luminosity indicator to constrain cosmological parameters and the transition redshift. Reference [18] made use of five luminosity indicators calibrated with 69 events by assuming two adopted cosmological models to construct the GRB Hubble diagram. Reference [23] suggested that the time variation of the dark energy is small or zero up to $z \sim 6$ using the $E_{p}-L$ relation. Reference [24] extended the Hubble diagram up to $z=5.6$ using 63 gamma-ray bursts (GRBs) via $E_{p}-L$ relation and found that these GRB data were consistent with the concordance model within $2 \sigma$ level. In a word, a lot of other works in this so-called GRB cosmology field have been published (please see $[19,25]$ for reviews). However, there is a so-called circularity problem in the calibration of these luminosity relations. Because of the current poor information on low$z$ GRBs, these luminosity relations necessarily depend on the assumed cosmology. Some authors attempted to circumvent the circularity problem by using a less model-dependent approach, such as the scatter method [26, 27], the luminosity distance method [28], the Bayesian method [29, 30], and the method by fitting relation parameters of GRBs and cosmological parameters simultaneously $[31,32]$. However, these statistical approaches still can not avoid the circularity problem completely, because a particular cosmology model is required in doing the joint fitting. This means that the parameters of the calibrated relations are still coupled to the cosmological parameters derived from a given cosmological model.
To solve the circularity problem completely, one should calibrate the GRB relations in a cosmology-independent way. Recently, a new method to calibrate GRBs in a cosmological model-independent way has been presented [33-35]. This method is very similar to the calibration for $\mathrm{SNe}$ Ia by measuring Cepheid variables in the same galaxy, and it is free from the circularity problem. Cepheid variables have been regarded as the first-order standard candles for calibrating $\mathrm{SNe}$ Ia which are the secondary standard candles. Similarly, if we regard $\mathrm{SNe}$ Ia as the first-order standard candles, we can also calibrate GRBs relations with a large number of $\mathrm{SNe}$ Ia since objects at the same redshift should have the same luminosity distance in any cosmology. This method is one of the interpolation procedures which obtain the distance moduli of GRBs in the redshift range of SNe Ia by interpolating from SNe Ia data in the Hubble diagram. Then, if we assume that the GRB luminosity relations do not evolve with redshift, we can extend the calibrated luminosity relations to high- $z$ and derive the distance moduli of high$z$ GRBs. From these obtained distance moduli, we can constrain the cosmological parameters.

In this paper, we will try to determine the cosmological parameters and dark energy using both the updated 139 GRBs and 580 SNe Ia. In Section 2, we will describe the data we will use and our method of calibration. To avoid any assumption on cosmological models, we will use the distance moduli of $580 \mathrm{SNe}$ Ia from the Union2.1 sample to calibrate five GRB luminosity relations in the redshift range of SNe Ia sample $(z<1.4)$. Then, the distance moduli of 82 high- $z$ GRBs $(z>$ 1.4) can be obtained from the five calibrated GRB luminosity relations. The joint constraints on the Cardassian expansion model and dark energy with 580 SNe and 82 calibrated GRBs' data whose $z>1.4$ will be presented in Section 3. Finally, we will summarize our findings and present a brief discussion.

\section{Calibrating the Updated Luminosity Relations of GRBs}

2.1. Observational Data and Methodology. As mentioned above, we calibrate the updated luminosity relations of GRBs using low- $z$ events whose distance moduli can be obtained by those of Type Ia supernovae. Actually, we use the cosmologyindependent calibration method developed by [33-35]. This method is one of the interpolation procedures which use the abundant SNe Ia sample to interpolate the distance moduli of GRBs in the redshift range of SNe Ia sample $(z<1.4)$. More recently, the Supernova Cosmology Project collaboration released their latest $\mathrm{SNe}$ Ia dataset known as the Union2.1 sample, which contains $580 \mathrm{SNe}$ detections [36]. Obviously, there are rich SNe Ia data points, and we can make a better interpolation by using this dataset.

Our updated GRB sample includes $139 \mathrm{GRB}$ s with redshift measurements; there are 57 GRBs at $z<1.4$ and 82 GRBs at $z>1.4$. This sample is shown in Table 1 , which includes the following information for each GRB: (1) its name; (2) the redshift; (3) the bolometric peak flux $P_{\text {bolo }}$; (4) the bolometric fluence $S_{\text {bolo }}$; (5) the beaming factor $f_{\text {beam }}$; $(6)$ the time lag $\tau_{\text {lag }}$; (7) the spectral peak energy $E_{p}$; and (8) the minimum rise time $\tau_{\mathrm{RT}}$. All of these data were obtained from previously 
TABLE 1: Luminosities and luminosity indicators.

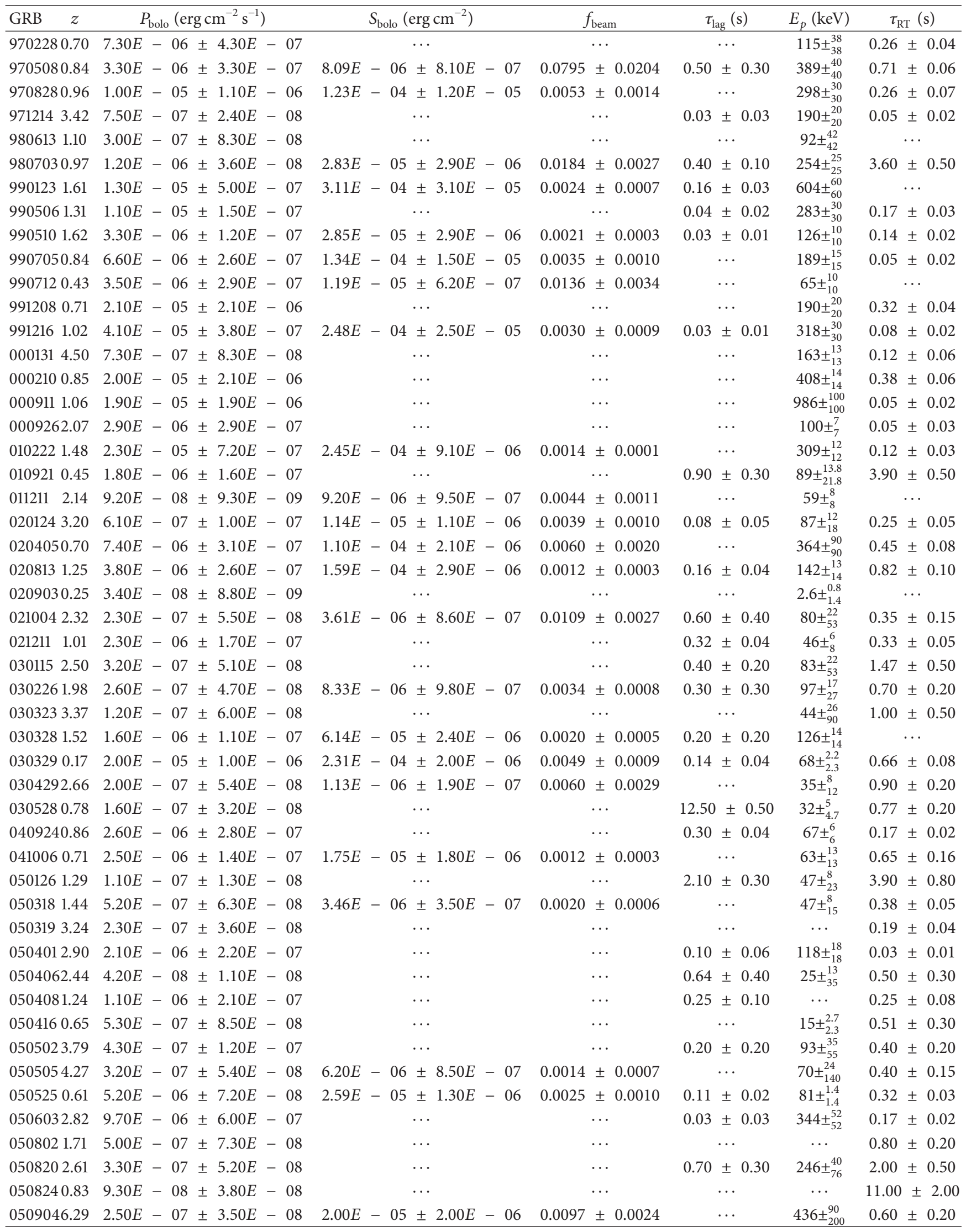


TABle 1: Continued.

\begin{tabular}{|c|c|c|c|c|c|c|c|c|c|c|c|c|c|}
\hline GRB & $z$ & & $P_{\text {bolo }}\left(\operatorname{erg~cm}^{-2} \mathrm{~s}^{-1}\right)$ & & & $S_{\text {bolo }}(\mathrm{er}$ & $\operatorname{erg~} \mathrm{cm}^{-2}$ ) & & & $f_{\text {beam }}$ & $\tau_{\text {lag }}(\mathrm{s})$ & $E_{p}(\mathrm{keV})$ & $\tau_{\mathrm{RT}}(\mathrm{s})$ \\
\hline 050908 & 3.35 & $80 E$ & $-08 \pm 1.50 E$ & -08 & & & $\cdots$ & & & $\ldots$ & $\ldots$ & $41 \pm_{9}^{5}$ & $1.50 \pm 0.30$ \\
\hline 050922 & 2.20 & $2.00 E$ & $-06 \pm 7.30 E$ & -08 & & & $\cdots$ & & & $\ldots$ & $0.06 \pm 0.02$ & $198 \pm_{38}^{22}$ & $0.13 \pm 0.02$ \\
\hline 051022 & 0.80 & $1.10 E$ & $-05 \pm 8.70 E$ & -07 & $40 E$ & $-04=$ & $\pm 1.20 E$ & -05 & 0029 & \pm 0.0001 & $\cdots$ & $510 \pm_{22}^{20}$ & $0.19 \pm 0.04$ \\
\hline 051109 & 2.35 & $7.80 E$ & $-07 \pm 9.70 E$ & -08 & & & $\cdots$ & & & $\ldots$ & $\cdots$ & $161 \pm_{130}^{35}$ & $1.30 \pm 0.40$ \\
\hline 051111 & 1.55 & $3.90 E$ & $-07 \pm 5.80 E$ & -08 & & & $\cdots$ & & & $\cdots$ & $1.02 \pm 0.10$ & $\cdots$ & $3.20 \pm 1.00$ \\
\hline 060108 & 2.03 & $1.10 E$ & $-07 \pm 1.10 E$ & -07 & & & $\cdots$ & & & $\ldots$ & $\cdots$ & $65 \pm_{600}^{10}$ & $0.40 \pm 0.20$ \\
\hline 060115 & 3.53 & $1.30 E$ & $-07 \pm 1.60 E$ & -08 & & & $\cdots$ & & & $\ldots$ & $\cdots$ & $62 \pm_{19}^{6}$ & $0.40 \pm 0.20$ \\
\hline 60116 & 6.60 & $2.00 E$ & $-07 \pm 1.10 E$ & -07 & & & .. & & & $\ldots$ & $\cdots$ & $139 \pm_{400}^{36}$ & $1.30 \pm 0.50$ \\
\hline 60124 & 2.30 & $1.10 E$ & $-06 \pm 1.20 E$ & -07 & $37 E$ & $-05=$ & $\pm 3.40 E$ & -06 & 21 & \pm 0 & $0.08 \pm 0.04$ & $237 \pm_{76}^{51}$ & $0.30 \pm 0.10$ \\
\hline 60206 & 4.05 & $4.40 E$ & $-07 \pm 1.90 E$ & -08 & & & $\cdots$ & & & $\ldots$ & $0.10 \pm 0.10$ & $75 \pm_{12}^{12}$ & $1.25 \pm 0.25$ \\
\hline 060210 & 3.91 & $5.50 E$ & $-07 \pm 2.20 E$ & -08 & $1.94 E$ & $-05=$ & $\pm 1.20 E$ & -06 & 0.0005 & \pm 0.0001 & $0.13 \pm 0.08$ & $149 \pm_{400}^{35}$ & $0.50 \pm 0.20$ \\
\hline 060223 & 4.41 & $2.10 E$ & $-07 \pm 3.70 E$ & -08 & & & $\cdots$ & & & $\cdots$ & $0.38 \pm 0.10$ & $71 \pm_{100}^{10}$ & $0.50 \pm 0.10$ \\
\hline 060418 & 1.49 & $1.50 E$ & $-06 \pm 5.90 E$ & -08 & & & $\cdots$ & & & $\ldots$ & $0.26 \pm 0.06$ & $230 \pm_{20}^{20}$ & $0.32 \pm 0.08$ \\
\hline 060502 & 1.51 & $3.70 E$ & $-07 \pm 1.60 E$ & -07 & & & $\cdots$ & & & $\ldots$ & $3.50 \pm 0.50$ & $156 \pm_{400}^{33}$ & $3.10 \pm 0.30$ \\
\hline 060510 & 4.90 & $1.00 E$ & $-07 \pm 1.70 E$ & -08 & & & $\cdots$ & & & $\ldots$ & $\cdots$ & $95 \pm_{60}^{30}$ & $\cdots$ \\
\hline 060526 & 3.21 & $2.40 E$ & $-07 \pm 3.30 E$ & -08 & $1.17 E$ & $-06=$ & $\pm 1.70 E$ & -07 & 34 & \pm 0 & $0.13 \pm 0.03$ & $25 \pm_{5}^{5}$ & $0.20 \pm 0.05$ \\
\hline 60604 & 2.68 & $9.00 E$ & $-08 \pm 1.60 E$ & -08 & & & $\cdots$ & & & $\ldots$ & $5.00 \pm 1.00$ & $40 \pm_{5}^{5}$ & $0.60 \pm 0.20$ \\
\hline 060605 & 3.80 & $1.20 E$ & $-07 \pm 5.50 E$ & -08 & & & .. & & & $\ldots$ & $5.00 \pm 3.00$ & $169 \pm_{200}^{30}$ & $2.00 \pm 0.50$ \\
\hline 060607 & 3.08 & $2.70 E$ & $-07 \pm 8.10 E$ & -08 & & & $\cdots$ & & & $\cdots$ & $2.00 \pm 0.5$ & $120 \pm_{190}^{17}$ & $2.00 \pm 0.20$ \\
\hline 060707 & 3.43 & $1.53 E$ & $-07 \pm 2.12 E$ & -08 & $3.41 E$ & $-06=$ & $\pm 1.96 E$ & -07 & & $\cdots$ & $\cdots$ & $63 \pm_{13}^{6}$ & $\cdots$ \\
\hline 060714 & 2.71 & $2.30 E$ & $-07 \pm 1.42 E$ & -08 & $6.88 E$ & $-06=$ & $\pm 2.47 E$ & -07 & & $\cdots$ & $\cdots$ & $03 \pm_{21}^{16}$ & $\cdots$ \\
\hline 660729 & 0.54 & $1.93 E$ & $-07 \pm 1.30 E$ & -08 & $6.43 E$ & $-06=$ & $\pm 3.16 E$ & -07 & & $\cdots$ & $\cdots$ & $61 \pm_{9}^{9}$ & $\cdots$ \\
\hline 060814 & 0.84 & $1.83 E$ & $-06 \pm 4.44 E$ & -08 & $4.94 E$ & $-05=$ & $\pm 4.91 E$ & -07 & & $\ldots$ & $0.29 \pm 0.03$ & $257 \pm \pm_{74}^{35}$ & $1.65 \pm 0.24$ \\
\hline 060904B & 30.70 & $4.37 E$ & $-07 \pm 2.28 E$ & -08 & $4.05 E$ & $-06=$ & $\pm 2.17 E$ & -07 & & $\ldots$ & $0.36 \pm 0.09$ & $80 \pm_{770}^{12}$ & $1.00 \pm 0.16$ \\
\hline 060908 & 2.43 & $6.69 E$ & $-07 \pm 3.36 E$ & -08 & $7.68 E$ & $-06=$ & $\pm 1.85 E$ & -07 & & $\ldots$ & $0.26 \pm 0.06$ & $151 \pm_{112}^{25}$ & $0.52 \pm 0.09$ \\
\hline 060926 & 3.21 & $1.56 E$ & $-07 \pm 1.22 E$ & -08 & $5.47 E$ & $-07=$ & $\pm 3.80 E$ & -08 & & $\cdots$ & $1.03 \pm 0.11$ & $20 \pm_{11}^{11}$ & $\cdots$ \\
\hline 60927 & 5.60 & $4.02 E$ & $-07 \pm 1.54 E$ & -08 & $E$ & $-06=$ & $\pm 8.67 E$ & -08 & & $\ldots$ & $0.12 \pm 0$ & $72 \pm_{15}^{7}$ & $0.46 \pm 0.12$ \\
\hline 061007 & 1.26 & $7.20 E$ & $-06 \pm 1.11 E$ & -07 & $2.24 E$ & $-04=$ & $\pm 1.72 E$ & -06 & & $\cdots$ & $0.11 \pm 0.01$ & $399 \pm_{12}^{11}$ & $0.38 \pm 0.02$ \\
\hline $61110 \mathrm{~A}$ & 0.76 & $9.79 E$ & $-08 \pm 1.35 E$ & -08 & $2.71 E$ & $-06=$ & $\pm 1.18 E$ & -07 & & $\cdots$ & $\cdots$ & $90 \pm_{13}^{13}$ & $\cdots$ \\
\hline 061110B & 3.44 & $1.79 E$ & $-07 \pm 2.66 E$ & -08 & $6.12 E$ & $-06=$ & $\pm 3.38 E$ & -07 & & $\ldots$ & $0.24 \pm 0.36$ & $517 \pm_{53}^{53}$ & $0.79 \pm 0.64$ \\
\hline 061121 & 1.31 & $8.04 E$ & $-06 \pm 1.07 E$ & -07 & $6.53 E$ & $-05=$ & $\pm 5.76 E$ & -07 & & $\ldots$ & $0.03 \pm 0.01$ & $606 \pm_{55}^{44}$ & $0.98 \pm 0.19$ \\
\hline 061222B & 3.36 & $2.29 E$ & $-07 \pm 3.15 E$ & -08 & $5.01 E$ & $-06=$ & $\pm 2.49 E$ & -07 & & $\cdots$ & $\cdots$ & $49 \pm_{8}^{8}$ & $\cdots$ \\
\hline 070110 & 2.35 & $1.12 E$ & $-07 \pm 1.36 E$ & -08 & $4.04 E$ & $-06=$ & $\pm 1.64 E$ & -07 & & $\cdots$ & $\cdots$ & $110 \pm_{30}^{30}$ & $\cdots$ \\
\hline 070208 & 1.17 & $1.39 E$ & $-07 \pm 2.06 E$ & -08 & $1.06 E$ & $-06=$ & $\pm 1.46 E$ & -07 & & $\cdots$ & $\cdots$ & $51 \pm_{10}^{10}$ & $\cdots$ \\
\hline 070318 & 0.84 & $4.10 E$ & $-07 \pm 2.12 E$ & -08 & $7.34 E$ & $-06=$ & $\pm 2.01 E$ & -07 & & $\ldots$ & $\cdots$ & $154 \pm_{19}^{19}$ & $0.72 \pm 0.2$ \\
\hline 70411 & 2.95 & $1.50 E$ & $-07 \pm 1.31 E$ & -08 & $6.29 E$ & $-06=$ & $\pm 2.19 E$ & -07 & & $\cdots$ & $\cdots$ & $83 \pm_{11}^{11}$ & $\cdots$ \\
\hline 70506 & 2.31 & $1.67 E$ & $-07 \pm 1.38 E$ & -08 & $5.16 E$ & $-07=$ & $\pm 3.43 E$ & -08 & & $\ldots$ & $2.52 \pm 0$. & $31 \pm_{2}^{3}$ & $0.12 \pm 0.06$ \\
\hline 70508 & 0.82 & $7.67 E$ & $-06 \pm 1.18 E$ & -07 & $7.26 E$ & $-05=$ & $\pm 6.15 E$ & -07 & & $\ldots$ & $0.04 \pm 0.01$ & $233 \pm_{7}^{7}$ & $0.20 \pm 0.01$ \\
\hline 070521 & 0.55 & $2.09 E$ & $-06 \pm 5.26 E$ & -08 & $2.97 E$ & $-05=$ & $\pm 4.00 E$ & -07 & & $\cdots$ & $0.04 \pm 0.01$ & $222 \pm_{16}^{12}$ & $0.58 \pm 0.06$ \\
\hline 070529 & 2.50 & $3.32 E$ & $-07 \pm 5.08 E$ & -08 & $7.44 E$ & $-06=$ & $\pm 4.31 E$ & -07 & & $\cdots$ & $\cdots$ & $180 \pm_{52}^{52}$ & $\cdots$ \\
\hline 070611 & 2.04 & $1.45 E$ & $-07 \pm 2.25 E$ & -08 & $9.52 E$ & $-07=$ & $\pm 8.44 E$ & -08 & & $\cdots$ & $\cdots$ & $92 \pm_{30}^{30}$ & $\cdots$ \\
\hline 070612A & 0.62 & $2.77 E$ & $-07 \pm 4.24 E$ & -08 & $2.72 E$ & $-05=$ & $\pm 9.37 E$ & -07 & & $\ldots$ & $\cdots$ & $87 \pm_{17}^{17}$ & $2.49 \pm 1.48$ \\
\hline 070714B & 0.92 & $3.24 E$ & $-06 \pm 1.46 E$ & -07 & $8.91 E$ & $-06=$ & $\pm 6.77 E$ & -07 & & $\cdots$ & $0.03 \pm 0.01$ & $1120 \pm_{473}^{230}$ & $0.45 \pm 0.04$ \\
\hline 70802 & 2.45 & $6.38 E$ & $-08 \pm 9.69 E$ & -09 & $6.50 E$ & $-07=$ & $\pm 7.05 E$ & -08 & & $\ldots$ & $\cdots$ & $70 \pm_{25}^{25}$ & $\cdots$ \\
\hline 070810A & 2.17 & $2.77 E$ & $-07 \pm 1.77 E$ & -08 & $1.59 E$ & $-06=$ & $\pm 8.43 E$ & -08 & & $\ldots$ & $1.09 \pm 0.23$ & $44 \pm_{9}^{9}$ & $0.73 \pm 0.22$ \\
\hline 071003 & 1.10 & $4.71 E$ & $-06 \pm 1.82 E$ & -07 & $6.73 E$ & $-05=$ & $\pm 1.48 E$ & -06 & & $\cdots$ & $0.38 \pm 0.05$ & $799 \pm_{75}^{61}$ & $0.88 \pm 0.07$ \\
\hline 071010A & 0.98 & $1.17 E$ & $-07 \pm 2.67 E$ & -08 & $4.97 E$ & $-07=$ & $\pm 6.05 E$ & -08 & & $\cdots$ & $\cdots$ & $27 \pm_{10}^{10}$ & $\cdots$ \\
\hline 071010B & 0.95 & $9.20 E$ & $-07 \pm 2.18 E$ & -08 & $8.37 E$ & $-06=$ & $\pm 1.16 E$ & -07 & & $\cdots$ & $0.84 \pm 0.04$ & $52 \pm_{6}^{8}$ & $1.21 \pm 0.03$ \\
\hline 071031 & 2.69 & $7.08 E$ & $-08 \pm 8.61 E$ & -09 & $2.19 E$ & $-06=$ & $\pm 1.92 E$ & -07 & & $\cdots$ & $\cdots$ & $24 \pm_{7}^{7}$ & $\cdots$ \\
\hline
\end{tabular}


TABLE 1: Continued.

\begin{tabular}{|c|c|c|c|c|c|c|c|}
\hline GRB & $z$ & $P_{\text {bolo }}\left(\mathrm{erg} \mathrm{cm}^{-2} \mathrm{~s}^{-1}\right)$ & $S_{\text {bolo }}\left(\operatorname{erg~cm}^{-2}\right)$ & $f_{\text {beam }}$ & $\tau_{\text {lag }}(\mathrm{s})$ & $E_{p}(\mathrm{keV})$ & $\tau_{\mathrm{RT}}(\mathrm{s})$ \\
\hline 071117 & 1.33 & $2.71 E-06 \pm 5.83 E-08$ & $7.97 E-06 \pm 2.02 E-07$ & $\cdots$ & $0.60 \pm 0.01$ & $278 \pm_{143}^{48}$ & $0.20 \pm 0.02$ \\
\hline 071122 & 1.14 & $6.76 E-08 \pm 2.06 E-08$ & $1.41 E-06 \pm 1.63 E-07$ & $\cdots$ & $\cdots$ & $73 \pm_{30}^{30}$ & $\cdots$ \\
\hline 080210 & 2.64 & $2.57 E-07 \pm 1.95 E-08$ & $4.17 E-06 \pm 1.41 E-07$ & $\cdots$ & $0.53 \pm 0.17$ & $73 \pm_{15}^{15}$ & $0.57 \pm 0.44$ \\
\hline 080310 & 2.43 & $1.83 E-07 \pm 1.72 E-08$ & $5.49 E-06 \pm 2.90 E-07$ & $\cdots$ & $\cdots$ & $28 \pm_{6}^{6}$ & $0.41 \pm 0.55$ \\
\hline 080319B & 0.94 & $1.55 E-05 \pm 1.91 E-07$ & $5.25 E-04 \pm 3.94 E-06$ & $\ldots$ & $0.02 \pm 0.01$ & $651 \pm_{8}^{8}$ & $0.14 \pm 0.01$ \\
\hline 080319C & 1.95 & $2.22 E-06 \pm 7.79 E-08$ & $1.77 E-05 \pm 2.99 E-07$ & $\ldots$ & $\cdots$ & $307 \pm_{85}^{56}$ & $0.21 \pm 0.12$ \\
\hline 080330 & 1.51 & $1.33 E-07 \pm 1.80 E-08$ & $8.77 E-07 \pm 1.26 E-07$ & $\cdots$ & $\cdots$ & $20 \pm_{9}^{9}$ & $\cdots$ \\
\hline 080411 & 1.03 & $1.04 E-05 \pm 1.31 E-07$ & $8.75 E-05 \pm 2.01 E-07$ & $\cdots$ & $0.21 \pm 0.01$ & $259 \pm_{21}^{16}$ & $0.65 \pm 0.01$ \\
\hline $080413 \mathrm{~A}$ & 2.43 & $1.22 E-06 \pm 2.65 E-08$ & $9.86 E-06 \pm 1.71 E-07$ & $\cdots$ & $0.13 \pm 0.03$ & $170 \pm_{48}^{24}$ & $0.23 \pm 0.03$ \\
\hline 080413B & 1.10 & $3.17 E-06 \pm 8.25 E-08$ & $8.00 E-06 \pm 1.52 E-07$ & $\cdots$ & $0.23 \pm 0.01$ & $73 \pm_{10}^{10}$ & $0.50 \pm 0.03$ \\
\hline 080430 & 0.77 & $4.60 E-07 \pm 2.15 E-08$ & $3.01 E-06 \pm 1.53 E-07$ & $\cdots$ & $0.68 \pm 0.08$ & $80 \pm_{15}^{15}$ & $0.76 \pm 0.12$ \\
\hline 080516 & 3.20 & $2.77 E-07 \pm 2.80 E-08$ & $5.88 E-07 \pm 5.50 E-08$ & $\cdots$ & $0.15 \pm 0.01$ & $66 \pm_{24}^{24}$ & $\cdots$ \\
\hline 080520 & 1.55 & $8.23 E-08 \pm 1.00 E-08$ & $1.59 E-07 \pm 3.00 E-08$ & $\cdots$ & $\cdots$ & $12 \pm_{5}^{5}$ & $\cdots$ \\
\hline 080603B & 2.69 & $7.57 E-07 \pm 2.63 E-08$ & $7.02 E-06 \pm 1.78 E-07$ & $\cdots$ & $0.08 \pm 0.01$ & $85 \pm_{55}^{18}$ & $0.22 \pm 0.03$ \\
\hline 080605 & 1.64 & $5.99 E-06 \pm 1.10 E-07$ & $4.72 E-05 \pm 4.32 E-07$ & $\cdots$ & $0.11 \pm 0.01$ & $246 \pm_{14}^{11}$ & $0.22 \pm 0.01$ \\
\hline 080607 & 3.04 & $8.35 E-06 \pm 2.42 E-07$ & $1.00 E-04 \pm 0.00 E+00$ & $\cdots$ & $0.04 \pm 0.01$ & $394 \pm_{35}^{33}$ & $0.18 \pm 0.06$ \\
\hline 080707 & 1.23 & $1.68 E-07 \pm 1.02 E-08$ & $1.26 E-06 \pm 8.87 E-08$ & $\cdots$ & $\cdots$ & $73 \pm_{20}^{20}$ & $\cdots$ \\
\hline 080721 & 2.60 & $9.57 E-06 \pm 5.01 E-07$ & $5.99 E-05 \pm 3.04 E-06$ & $\cdots$ & $0.13 \pm 0.05$ & $485 \pm_{41}^{36}$ & $0.09 \pm 0.04$ \\
\hline 080810 & 3.35 & $9.76 E-07 \pm 8.40 E-08$ & $1.80 E-05 \pm 1.30 E-06$ & $\ldots$ & $\ldots$ & $313.5 \pm_{73.6}^{73.6}$ & $\cdots$ \\
\hline 080913 & 6.70 & $2.31 E-07 \pm 4.00 E-08$ & $1.15 E-06 \pm 1.20 E-07$ & $\ldots$ & .. & $93.1 \pm_{56.1}^{56.1}$ & $\cdots$ \\
\hline 080916A & 0.69 & $1.06 E-06 \pm 1.60 E-07$ & $2.19 E-05 \pm 7.30 E-06$ & $\cdots$ & $\cdots$ & $109 \pm_{9}^{9}$ & $\cdots$ \\
\hline 081121 & 2.51 & $1.87 E-06 \pm 4.80 E-07$ & $1.73 E-05 \pm 3.20 E-06$ & $\cdots$ & $\cdots$ & $248 \pm_{38}^{32}$ & . \\
\hline 081222 & 2.77 & $2.62 E-06 \pm 3.80 E-07$ & $1.70 E-05 \pm 1.40 E-06$ & $\cdots$ & $\cdots$ & $134 \pm_{9}^{9}$ & $\cdots$ \\
\hline 090102 & 1.55 & $3.90 E-06 \pm 5.60 E-07$ & $3.66 E-05 \pm 3.40 E-06$ & $\cdots$ & $\cdots$ & $451 \pm_{73}^{58}$ & $\cdots$ \\
\hline 090323 & 3.57 & $3.80 E-06 \pm 6.90 E-07$ & $1.48 E-04 \pm 2.00 E-05$ & $\cdots$ & $\cdots$ & $416 \pm_{76}^{73}$ & $\cdots$ \\
\hline 090328 & 0.74 & $7.28 E-06 \pm 5.20 E-07$ & $1.37 E-04 \pm 8.00 E-06$ & $\cdots$ & $\cdots$ & $653 \pm_{45}^{45}$ & . \\
\hline 090423 & 8.20 & $3.24 E-07 \pm 6.80 E-08$ & $1.17 E-06 \pm 3.20 E-07$ & $\cdots$ & $\cdots$ & $82 \pm_{15}^{15}$ & . \\
\hline 090424 & 0.54 & $1.80 E-05 \pm 1.20 E-06$ & $5.85 E-05 \pm 2.10 E-06$ & $\cdots$ & $\cdots$ & $177 \pm_{3}^{3}$ & . \\
\hline 090516 & 4.11 & $5.93 E-07 \pm 3.40 E-08$ & $1.09 E-05 \pm 1.60 E-06$ & $\cdots$ & $\cdots$ & $185.6 \pm \pm_{98}^{42 .}$ & $\cdots$ \\
\hline 090618 & 0.54 & $8.58 E-06 \pm 9.60 E-07$ & $3.39 E-04 \pm 2.50 E-05$ & $\cdots$ & $\cdots$ & $155.5 \pm_{11.1}^{10.5}$ & $\cdots$ \\
\hline 090715B & 3.00 & $8.96 E-07 \pm 2.49 E-07$ & $1.09 E-05 \pm 1.60 E-06$ & $\cdots$ & $\cdots$ & $134 \pm_{56}^{30}$ & $\cdots$ \\
\hline 090812 & 2.45 & $2.17 E-06 \pm 2.40 E-07$ & $3.15 E-05 \pm 4.10 E-06$ & $\ldots$ & $\cdots$ & $572 \pm_{251}^{159}$ & $\ldots$ \\
\hline 090902B & 1.82 & $2.72 E-05 \pm 3.00 E-07$ & $3.78 E-04 \pm 3.00 E-06$ & $\cdots$ & $\cdots$ & $775 \pm_{11}^{11}$ & $\cdots$ \\
\hline 090926 & 2.11 & $1.82 E-05 \pm 3.00 E-07$ & $1.51 E-04 \pm 7.70 E-06$ & $\cdots$ & $\cdots$ & $314 \pm_{4}^{4}$ & $\cdots$ \\
\hline 090926B & 1.24 & $5.55 E-07 \pm 7.90 E-08$ & $1.66 E-05 \pm 5.00 E-07$ & $\cdots$ & $\cdots$ & $78.3 \pm_{7}^{7}$ & $\cdots$ \\
\hline 091018 & 0.97 & $1.82 E-06 \pm 8.40 E-07$ & $4.33 E-06 \pm 7.60 E-07$ & $\cdots$ & $\cdots$ & $19.2 \pm_{18}^{11}$ & $\cdots$ \\
\hline 091020 & 1.71 & $1.63 E-06 \pm 2.00 E-07$ & $1.68 E-05 \pm 3.70 E-06$ & $\cdots$ & $\cdots$ & $47.9 \pm_{7.1}^{7.1}$ & $\cdots$ \\
\hline 091029 & 2.75 & $2.82 E-07 \pm 1.60 E-08$ & $5.84 E-06 \pm 4.70 E-07$ & $\cdots$ & $\cdots$ & $61.4 \pm_{17.5}^{17.5}$ & $\cdots$ \\
\hline 091127 & 0.49 & $4.03 E-06 \pm 1.70 E-07$ & $2.65 E-05 \pm 5.00 E-07$ & $\cdots$ & $\cdots$ & $36 \pm_{2}^{2}$ & $\cdots$ \\
\hline 091208B & 1.06 & $3.47 E-06 \pm 6.00 E-07$ & $7.78 E-06 \pm 8.80 E-07$ & $\cdots$ & $\cdots$ & $124 \pm_{20.1}^{19.4}$ & $\cdots$ \\
\hline
\end{tabular}

published studies. Before GRB 060607, we take all the data directly from [18]. We adopt the data between GRB 060707 and GRB 080721 from [19]. For those GRBs detected after July 7, 2008, we adopt the data directly from [37]. Applying the interpolation method, we can derive the distance moduli of 57 low- $z$ GRBs and calibrate five GRB luminosity relations with this low- $z$ sample, that is, the $\tau_{\mathrm{lag}}-L$ relation, the $E_{p}-L$ relation, the $E_{p}-E_{\gamma}$ relation, the $\tau_{\mathrm{RT}}-L$ relation, and the
$E_{p}-E_{\text {iso }}$ relation. The isotropic peak luminosity of a burst is calculated by

$$
L=4 \pi D_{L}^{2} P_{\text {bolo }},
$$

the isotropic equivalent gamma-ray energy is given by

$$
E_{\text {iso }}=4 \pi D_{L}^{2} S_{\text {bolo }}(1+z)^{-1} \text {, }
$$


TABLE 2: Best-fitting results.

\begin{tabular}{lcccc}
\hline Relation & $a$ & $b$ & $N$ & $r$ \\
\hline$\tau_{\text {lag }}-L$ & $52.32 \pm 0.03$ & $-1.10 \pm 0.05$ & 27 & -0.75 \\
$E_{p}-L$ & $52.08 \pm 0.02$ & $1.60 \pm 0.04$ & 55 & 0.79 \\
$E_{p}-E_{\gamma}$ & $50.57 \pm 0.01$ & $1.76 \pm 0.03$ & 12 & 0.95 \\
$\tau_{\mathrm{RT}}-L$ & $52.85 \pm 0.03$ & $-1.51 \pm 0.06$ & 40 & -0.66 \\
$E_{p}-E_{\text {iso }}$ & $52.81 \pm 0.02$ & $1.53 \pm 0.04$ & 42 & 0.73 \\
\hline
\end{tabular}

and the collimation-corrected energy is

$$
E_{\gamma}=E_{\text {iso }} f_{\text {beam }}=4 \pi D_{L}^{2} S_{\text {bolo }} f_{\text {beam }}(1+z)^{-1} .
$$

Here, $D_{L}$ is the luminosity distance of the burst, $P_{\text {bolo }}$ and $S_{\text {bolo }}$ are the bolometric peak flux and fluence of gamma-rays, respectively, while $f_{\text {beam }}=\left(1-\cos \theta_{\text {jet }}\right)$ is the beaming factor, and $\theta_{\text {jet }}$ is the jet half-opening angle. We assume each GRB has bipolar jets, and $E_{\gamma}$ is the true energy of the bipolar jets.

For convenience, the luminosity relations involved in this paper can be generally written in the power-law forms:

$$
\log y=a+b \log x
$$

where $a$ and $b$ are the intercept and slope of the relation, respectively; $y$ is the luminosity ( $L$ in units of $\mathrm{erg} \mathrm{s}^{-1}$ ) or energy ( $E_{\text {iso }}$ or $E_{\gamma}$ in units of erg); $x$ is the GRB parameters measured in the rest frame, for example, $\tau_{\text {lag }}(1+z)^{-1} /(0.1 \mathrm{~s})$, $E_{p}(1+z) /(300 \mathrm{keV}), E_{p}(1+z) /(300 \mathrm{keV}), \tau_{\mathrm{RT}}(1+z)^{-1} /(0.1 \mathrm{~s})$, and $E_{p}(1+z) /(300 \mathrm{keV})$, for the 5 two-variable relations above.

2.2. Calibration. First of all, we obtain the distance moduli of 57 low- $z(z<1.4)$ GRBs by using cubic spline interpolation from the 580 Union2.1 SNe Ia compiled in [36]. The interpolated distance moduli $\mu$ of these 57 GRBs and their corresponding errors $\sigma_{\mu}$ are shown in Figure 1(a). The SNe Ia data are also plotted in Figure 1(a) for comparison. When the cubic spline interpolation is used, the error of the interpolated distance moduli $\mu$ for the GRB at redshift $z$ can be calculated by

$$
\sigma_{\mu}=\left[\left(\frac{z_{i+1}-z}{z_{i+1}-z_{i}}\right)^{2} \epsilon_{\mu, i}^{2}+\left(\frac{z-z_{i}}{z_{i+1}-z_{i}}\right)^{2} \epsilon_{\mu, i+1}^{2}\right]^{1 / 2},
$$

where $\epsilon_{\mu, i}$ and $\epsilon_{\mu, i+1}$ are errors of the SNe at nearby redshifts $z_{i}$ and $z_{i+1}$, respectively. With $D_{L}$ in units of $\mathrm{Mpc}$, the predicted distance modulus is defined as

$$
\mu=5 \log \left(D_{L}\right)+25 .
$$

Having estimated the distance moduli $\mu$ of 57 low- $z$ GRBs in a model-independent way, we can convert $\mu$ into luminosity distance $D_{L}$ by using (6). From (1)-(3) with the corresponding $P_{\text {bolo }}, S_{\text {bolo }}$, and $f_{\text {beam }}$, we can calculate $L, E_{\text {iso }}$, and $E_{\gamma}$. In Figures 1(b)-1(f), with the interpolation results, we show the five luminosity indicators for these 57 GRBs at $z<1.4$. For each relation, we perform a linear least-squares fit, taking into account both the $x$-axis error and the $y$-axis error. We also measure the scatter of each relation with the distance of the data points from the best-fit line, as done by [38]. The bestfitting results of the intercept $a$ and the slope $b$ with their $1 \sigma$ uncertainties and the linear correlation coefficients for each relation are summarized in Table 2 . The best-fitting results derived by using the interpolation method are carried out with these 57 GRBs at $z<1.4$. In other words, the results are derived by using data from $27,55,12,40$, and 42 GRBs for the $\tau_{\text {lag }}-L, E_{p}-L, E_{p}-E_{\gamma}, \tau_{\mathrm{RT}}-L$, and $E_{p}-E_{\text {iso }}$ relations, respectively.

Reference [19] found no statistically significant evidence for the redshift evolution of the luminosity relations. If the GRB luminosity relations indeed do not evolve with redshift, we can extend the calibrated luminosity relations to high- $z$ $(z>1.4)$ and derive the luminosity $(L)$ or energy $\left(E_{\text {iso }}\right.$ or $\left.E_{\gamma}\right)$ of each burst at high- $z$ by utilizing the calibrated relations. Therefore, the luminosity distance $D_{L}$ can be derived from (1)-(3). The uncertainty of the value of the luminosity or energy deduced from each relation is

$$
\sigma_{\log y}^{2}=\sigma_{a}^{2}+\left(\sigma_{b}^{2} \log x\right)^{2}+\left(\frac{b}{\ln 10} \frac{\sigma_{x}}{x}\right)^{2}+\sigma_{\text {int }}^{2},
$$

where $\sigma_{a}, \sigma_{b}$, and $\sigma_{x}$ are $1 \sigma$ uncertainties of the intercept $a$, the slope $b$, and the GRB measurable parameters $x$ and $\sigma_{\text {int }}$ is the systematic error in the fitting that accounts for the extra scatter of the luminosity relations. Then, we obtain the distance moduli $\mu$ for these 82 GRBs at $z>1.4$ using (6). The propagated uncertainties will depend on whether $P_{\text {bolo }}$ or $S_{\text {bolo }}$ are given by

$$
\sigma_{\mu}=\left[\left(\frac{5}{2} \sigma_{\log L}\right)^{2}+\left(\frac{5}{2 \ln 10} \frac{\sigma_{P_{\text {bolo }}}}{P_{\text {bolo }}}\right)^{2}\right]^{1 / 2},
$$

or

$$
\begin{gathered}
\sigma_{\mu}=\left[\left(\frac{5}{2} \sigma_{\log E_{\text {iso }}}\right)^{2}+\left(\frac{5}{2 \ln 10} \frac{\sigma_{S_{\text {bolo }}}}{S_{\text {bolo }}}\right)^{2}\right]^{1 / 2}, \\
\sigma_{\mu}=\left[\left(\frac{5}{2} \sigma_{\log E_{\gamma}}\right)^{2}+\left(\frac{5}{2 \ln 10} \frac{\sigma_{S_{\text {bolo }}}}{S_{\text {bolo }}}\right)^{2}\right. \\
\left.+\left(\frac{5}{2 \ln 10} \frac{\sigma_{f_{\text {beam }}}}{f_{\text {beam }}}\right)^{2}\right]^{1 / 2} .
\end{gathered}
$$

Here we ignore the uncertainty of $z$ in our calculations.

After obtaining the distance modulus of each GRB using one of these relations, we use the same method as [18] to calculate the real distance modulus, which is the weighted 


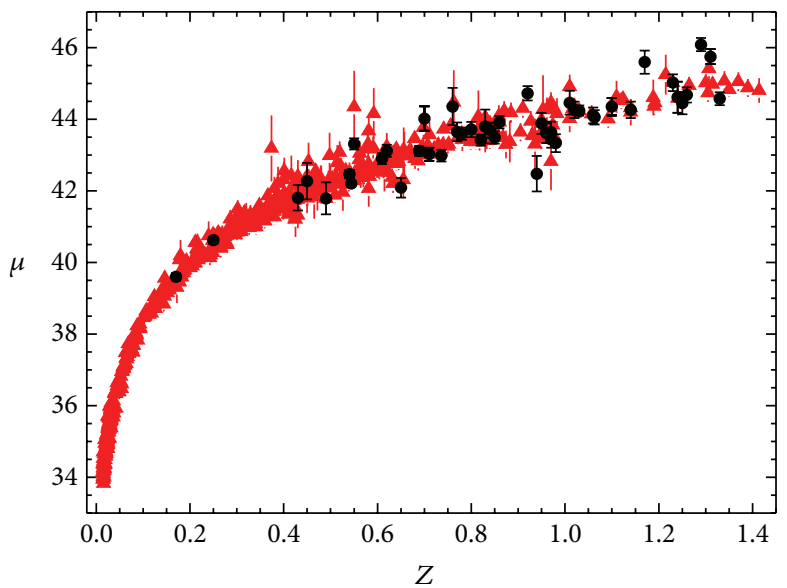

(a)

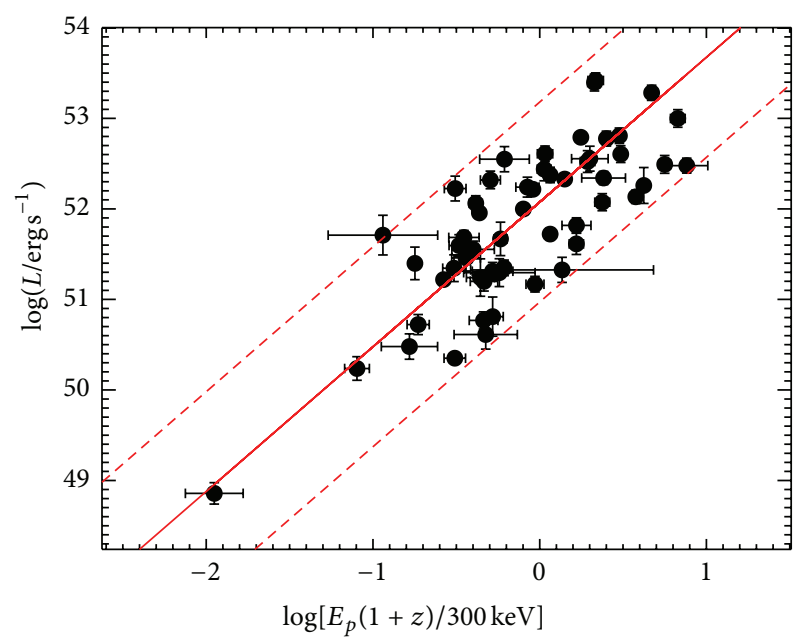

(c)

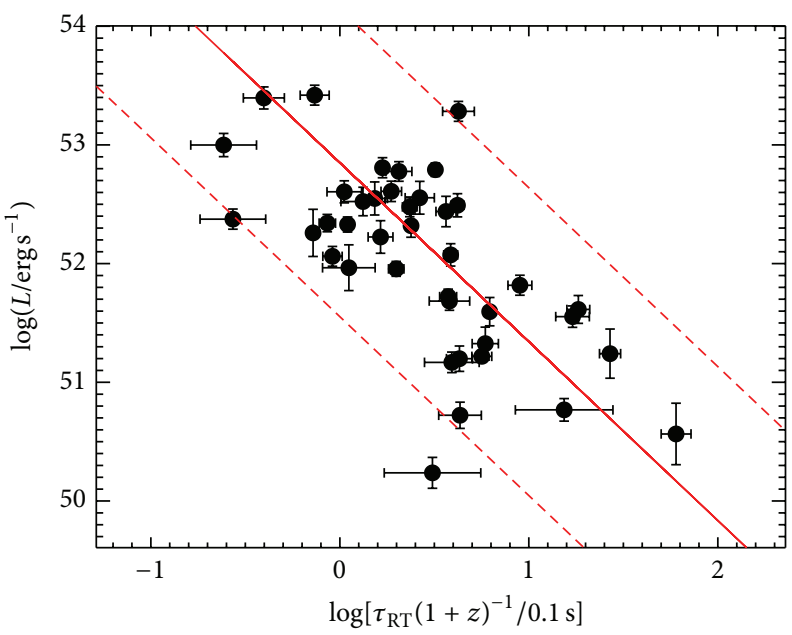

(e)

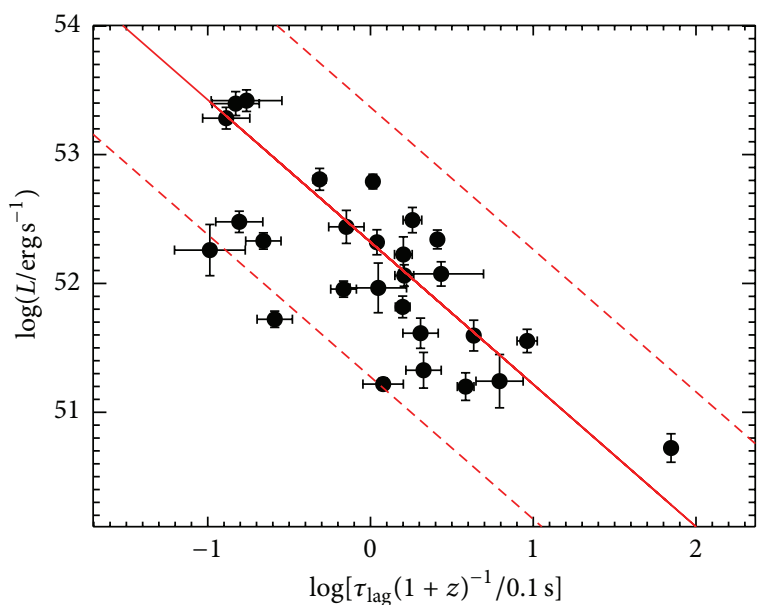

(b)

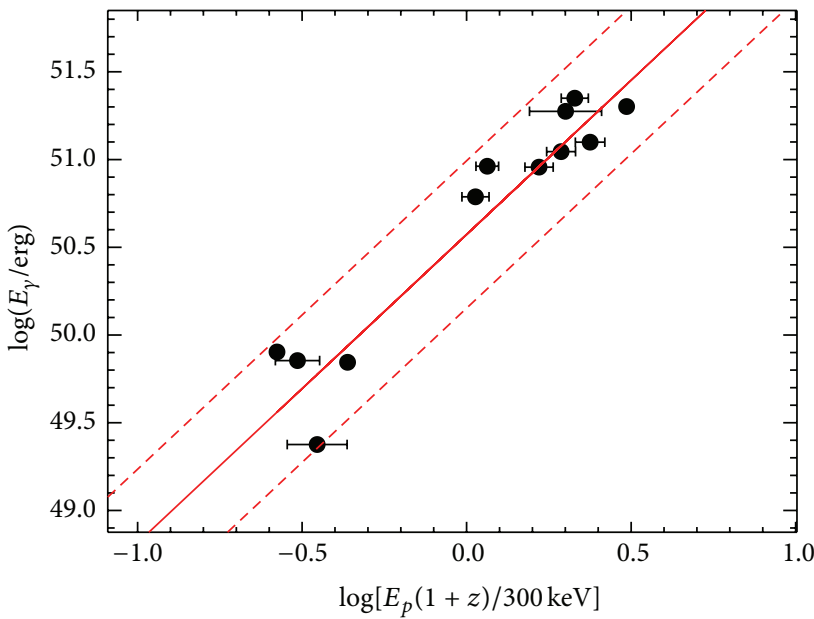

(d)

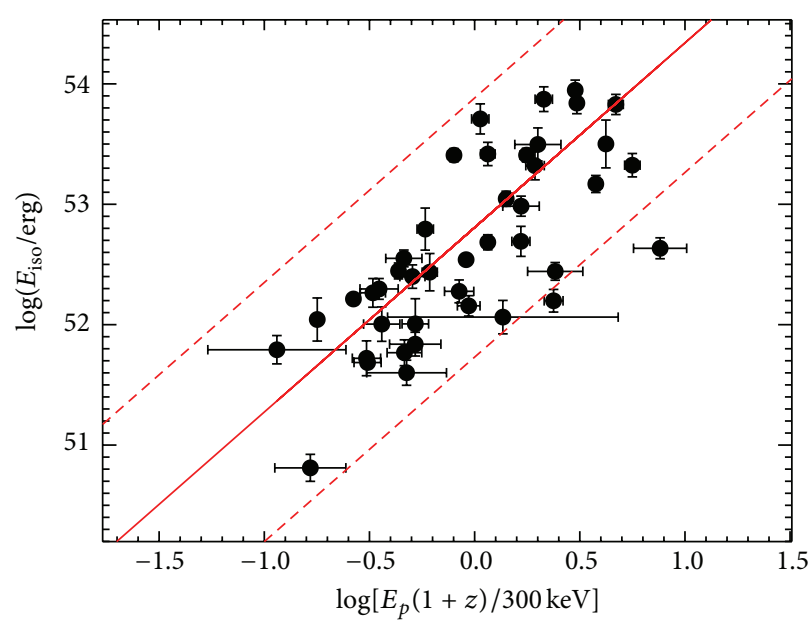

(f)

Figure 1: (a) The Hubble diagram of 580 SNe Ia (red triangles) and 57 GRBs at $z \leq 1.4$ (black dots) whose distance moduli are derived by using cubic spline interpolation. (b)-(f) The $\tau_{\text {lag }}-L, E_{p}-L, E_{p}-E_{\gamma}, \tau_{\mathrm{RT}}-L$, and $E_{p}-E_{\mathrm{iso}}$ correlations. The five correlations are calibrated with the sample at $z \leq 1.4$ using cubic spline interpolation. The solid lines show the best-fitting results, while the dashed lines represent their $2 \sigma$ dispersion around the best fits. 


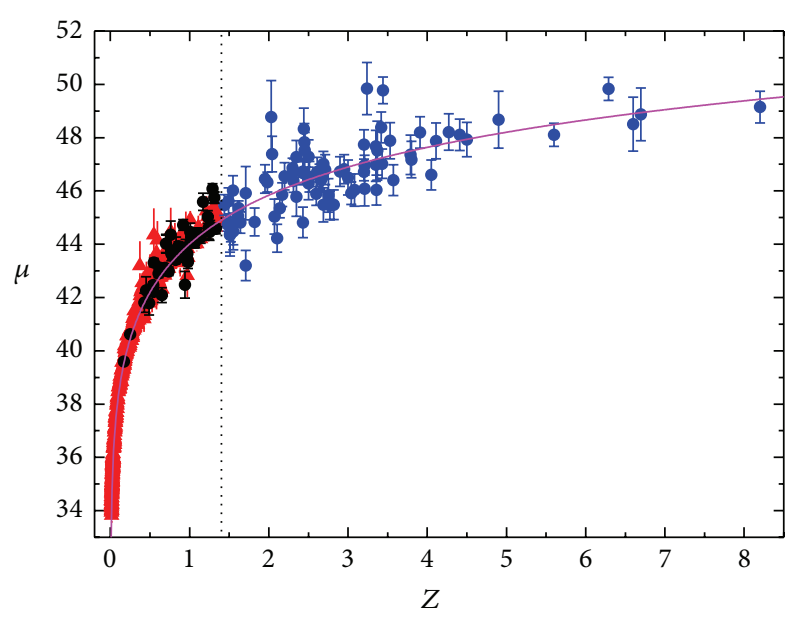

Figure 2: Hubble diagram of 580 SNIa (red triangles) and 139 GRBs (dots) obtained using the interpolation method. The 57 GRBs at $z \leq 1.4$ are obtained by interpolating from $\mathrm{SNe}$ Ia data (black dots); and the 82 GRBs at $z>1.4$ (blue dots) are obtained with the five correlations calibrated with the sample at $z \leq 1.4$ using the cubic spline interpolation method. The vertical dotted line represents $z=$ 1.4. The solid curve represents the best-fit cosmology for a flat $\Lambda \mathrm{CDM}$ universe: $\Omega_{m}=0.29, \Omega_{\Lambda}=0.71$.

average of all available distance moduli. The real distance modulus for each burst is

$$
\mu_{\text {fit }}=\frac{\sum_{i} \mu_{i} / \sigma_{\mu_{i}}^{2}}{\sum_{i} \sigma_{\mu_{i}}^{-2}}
$$

with its corresponding uncertainty $\sigma_{\mu_{\mathrm{fit}}}=\left(\sum_{i} \sigma_{\mu_{i}}^{-2}\right)^{-1 / 2}$, where the summation runs from 1 to 5 over the relations with available data and $\mu_{i}$ and $\sigma_{\mu_{i}}$ are the best estimated distance modulus and its corresponding uncertainty from the $i$ th relation.

Figure 2 shows the Hubble diagram from the Union2.1 SNe Ia sample and 139 GRBs. The combined Hubble diagram is consistent with the concordance cosmology. The 57 GRBs at $z<1.4$ are obtained using interpolation method directly from SNe data. The $82 \mathrm{GRBs}$ at $z>1.4$ are obtained by utilizing the five relations calibrated with the sample at $z<$ 1.4 using the cubic spline interpolation method.

\section{Constraints from Supernovae and GRBs}

The latest Type Ia SNe dataset known as the Union2.1 sample was recently released by the Supernova Cosmology Project collaboration, which contains $580 \mathrm{SNe}$ detections (see [36]). With luminosity distance $D_{L}(\xi, z)$ in units of Mpc (where $\xi$ stands for all the cosmological parameters that define the fitted model), the theoretical distance modulus $\mu_{\text {th }}$ can be calculated by using (6). The likelihood functions can be determined from the $\chi^{2}$ statistic:

$$
\chi_{\mathrm{SNe}}^{2}=\sum_{i=1}^{N} \frac{\left[\mu_{\mathrm{th}}\left(z_{i}\right)-\mu_{\mathrm{obs}}\left(z_{i}\right)\right]^{2}}{\sigma_{\mathrm{lc}}^{2}+\sigma_{\mathrm{ext}}^{2}+\sigma_{\mathrm{sample}}^{2}},
$$

where $\sigma_{\mathrm{lc}}$ is the propagated error from the covariance matrix of the light-curve fit and $\mu_{\mathrm{obs}}$ is the observational distance modulus. The uncertainties due to host galaxy peculiar velocities, Galactic extinction corrections, and gravitational lensing are included in $\sigma_{\text {ext }}$, and $\sigma_{\text {sample }}$ is a floating dispersion term containing sample-dependent systematic errors. The confidence regions can be found through marginalizing the likelihood functions over Hubble constant $H_{0}$ (i.e., integrating the probability density $p \propto \exp \left(-\chi^{2} / 2\right)$ for all values of $H_{0}$ ).

Gamma-ray bursts (GRBs) are the most luminous transient events in the cosmos. Owing to their high luminosity, GRBs can be detected out to the edge of the visible universe, constituting a powerful tool for constructing a Hubble diagram at high- $z$. We use the above calibration results obtained by using the interpolation methods directly from SNe Ia data. The $\chi^{2}$ value for the $82 \mathrm{GRBs}$ at $z>1.4$ is given by

$$
\chi_{\mathrm{GRB}}^{2}=\sum_{i=1}^{N} \frac{\left[\mu_{\mathrm{th}}\left(z_{i}\right)-\mu_{\mathrm{fit}, i}\right]^{2}}{\sigma_{\mu_{\mathrm{fti}, i}}^{2}},
$$

where $\mu_{\mathrm{fit}, i}$ and $\sigma_{\mu_{\mathrm{fit}, i}}$ are the fitted distance modulus and its error for each burst. We also marginalize the nuisance parameter $H_{0}$.

Motivated by these significant updates in the observations of SNe Ia and GRBs, it is natural to consider the joint constraints on cosmological parameters and dark energy with the latest observational data. We combine SNe Ia and GRBs by multiplying the likelihood functions. The total $\chi^{2}$ value is

$$
\chi_{\text {total }}^{2}=\chi_{\mathrm{SNe}}^{2}+\chi_{\mathrm{GRB}}^{2} .
$$

The best-fitting values of cosmological model are obtained by minimizing $\chi_{\text {total }}^{2}$.

3.1. $\Lambda C D M$ Model. In a Friedmann-Robertson-Walker (FRW) cosmology with mass density $\Omega_{m}$ and vacuum energy density $\Omega_{\Lambda}$, the luminosity distance is given as

$$
\begin{aligned}
D_{L}(z)= & \frac{c}{H_{0}} \frac{(1+z)}{\sqrt{\left|\Omega_{k}\right|}} \\
& \cdot \operatorname{sinn}\left\{\left|\Omega_{k}\right|^{1 / 2} \int_{0}^{z} \frac{d z}{\sqrt{\Omega_{m}(1+z)^{3}+\Omega_{\Lambda}+\Omega_{k}(1+z)^{2}}}\right\},
\end{aligned}
$$

where $c$ is the speed of light, $H_{0}$ is the Hubble constant at the present time, $\Omega_{k}=1-\Omega_{m}-\Omega_{\Lambda}$ represents the spatial curvature of the universe, and sinn is sinh when $\Omega_{k}>0$ and $\sin$ when $\Omega_{k}<0$. For a flat universe with $\Omega_{k}=0$, (14) simplifies to the form $(1+z) c / H_{0}$ times the integral. In this $\Lambda \mathrm{CDM}$ model, the transition redshift satisfies

$$
z_{T}=\left(\frac{2 \Omega_{\Lambda}}{\Omega_{m}}\right)^{1 / 3}-1 .
$$

We use the datasets discussed above to constrain cosmological parameters. In Figure 3(a), we show the confidence 


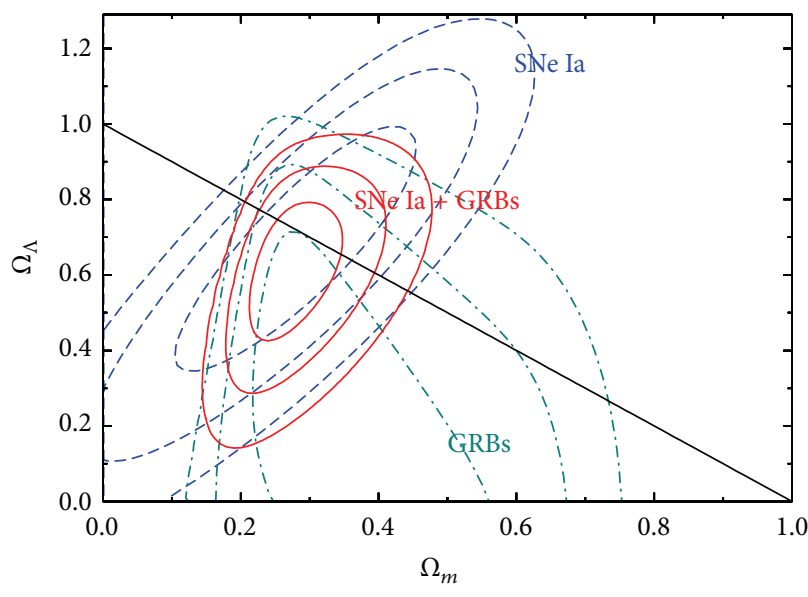

(a)

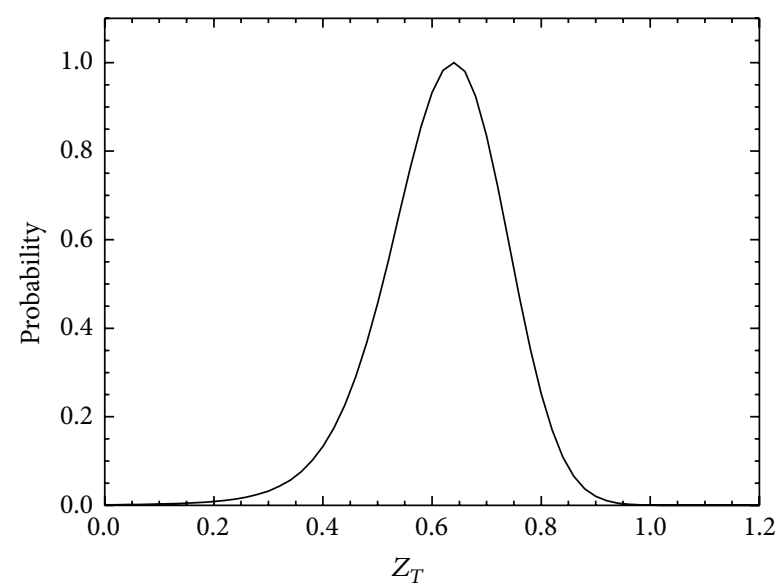

(b)

FIGURE 3: (a) The contour confidence levels of $\left(\Omega_{m}, \Omega_{\Lambda}\right)$ in the $\Lambda$ CDM model from the data for 82 GRBs $(z>1.4)$ (dark cyan dash-dotted lines), $580 \mathrm{SNe}$ Ia (blue dashed lines), and $82 \mathrm{GRBs}+580 \mathrm{SNe}$ Ia (red solid lines), respectively. The contours correspond to 1,2 , and $3 \sigma$ confidence regions. (b) The probability versus the transition redshift derived from the GRB and SNe Ia sample.

regions for $\left(\Omega_{m}, \Omega_{\Lambda}\right)$ from 82 GRBs (dark cyan dash-dotted lines), 580 SNe Ia (blue dotted lines), and 82 GRBs +580 SNe Ia (red solid lines), respectively. We can see that adding 82 high redshift GRBs $(z>1.4)$ to 580 SNe Ia $(z<1.4)$ significantly improves the constraint on $\Omega_{m}-\Omega_{\Lambda}$ plane. The $1 \sigma$ confidence region from all the datasets is $\left(\Omega_{m}, \Omega_{\Lambda}\right)=$ $\left(0.27_{-0.06}^{+0.08}, 0.62_{-0.19}^{+0.18}\right)$ with $\chi_{\min }^{2}=727.01$ for 659 degrees of freedom. Under the assumption of a flat universe (solid line), the contours yield $\left(\Omega_{m}, \Omega_{\Lambda}\right)=\left(0.29_{-0.04}^{+0.04}, 0.71_{-0.04}^{+0.04}\right)$. The transition redshift at which the universe switched from deceleration to acceleration phase is $z_{T}=0.64_{-0.14}^{+0.08}$ at the $1 \sigma$ confidence level (Figure 3(b)).

3.2. Cardassian Expansion Model. Reference [6] proposed the Cardassian expansion model as a possible alternative for explaining the acceleration of the universe that invokes no vacuum energy. This model allows an acceleration in a flat, matter-dominated cosmology. If we consider a spatially flat FRW universe, the Friedmann equation is modified as

$$
H^{2}=\frac{8 \pi G}{3}\left(\rho+C \rho^{n}\right)
$$

This modification may arise as a consequence of embedding our observable universe as a $(3+1)$-dimensional brane in extra dimensions or the self-interaction of dark matter. The luminosity distance in this model is given by

$$
\begin{aligned}
D_{L}(z)= & c H_{0}^{-1}(1+z) \\
& \cdot \int_{0}^{z} d z\left[(1+z)^{3} \Omega_{m}+\left(1-\Omega_{m}\right)(1+z)^{3 n}\right]^{-1 / 2} .
\end{aligned}
$$

Figure 4 shows constraints on $\Omega_{m}$ and $n$ from $1 \sigma$ to $3 \sigma$ confidence regions by fitting observational data. The dark cyan dash-dotted lines and blue dashed lines represent the results from $82 \mathrm{GRBs}$ and $580 \mathrm{SNe}$ Ia, respectively. The red

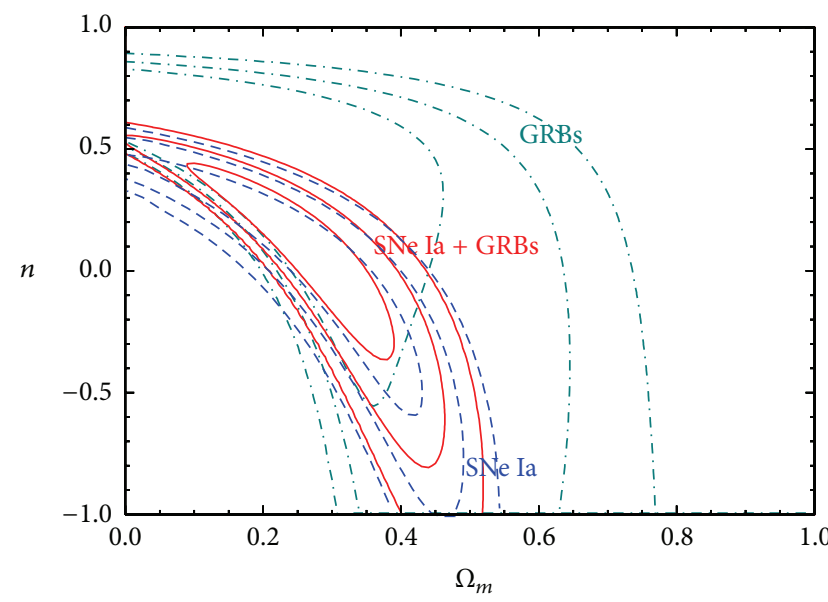

FIgURE 4: The contour confidence levels of $\left(\Omega_{m}, n\right)$ in the Cardassian expansion model from the data for 82 GRBs $(z>1.4)$ (dark cyan dash-dotted lines), 580 SNe Ia (blue dashed lines), and 82 GRBs + $580 \mathrm{SNe}$ Ia (red solid lines), respectively. The contours correspond to 1,2 , and $3 \sigma$ confidence regions.

solid contours show the constraints from the combination of these data. The best values are $\Omega_{m}=0.24_{-0.15}^{+0.15}$ and $n=$ $0.16_{-0.52}^{+0.30}$ at the $1 \sigma$ confidence level with $\chi_{\min }^{2}=727.31$ for 659 degrees of freedom. This result is consistent with the $\Lambda \mathrm{CDM}$ cosmology $(n=0)$ in the $1 \sigma$ confidence region.

3.3. $w(z)=w_{0}$ Model: Constant Equation of State. For the dark energy model with a constant equation of state $(w(z)=$ $\left.w_{0}\right)$, the luminosity distance for a flat universe is [39]

$$
\begin{aligned}
D_{L}(z)= & c H_{0}^{-1}(1+z) \\
& \cdot \int_{0}^{z} d z\left[(1+z)^{3} \Omega_{m}+\left(1-\Omega_{m}\right)(1+z)^{3\left(1+w_{0}\right)}\right]^{-1 / 2},
\end{aligned}
$$




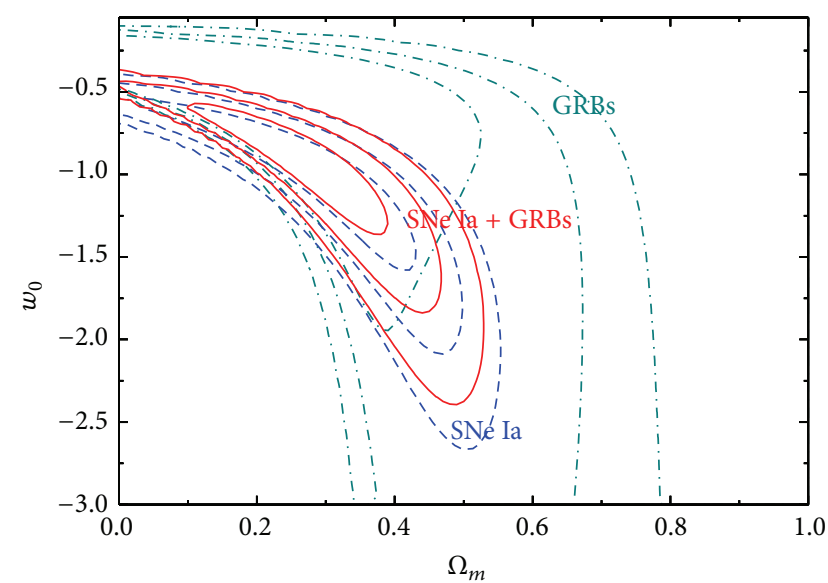

FIgURE 5: Constraints on $\Omega_{m}$ and $w_{0}$ from $1 \sigma$ to $3 \sigma$ confidence regions with dark energy whose equation state is constant. The contours are derived from GRBs (dark cyan dash-dotted lines), SNe Ia (blue dashed lines), and SNe Ia + GRBs (red solid lines), respectively.

and then the likelihood function depends on $\Omega_{m}$ and $w_{0}$. Figure 5 shows the likelihood contours on $\left(\Omega_{m}, w_{0}\right)$ plane for GRBs (dark cyan dash-dotted lines), SNe Ia (blue dashed lines), and SNe Ia + GRBs (red solid lines), respectively. The contours correspond to 1,2 , and $3 \sigma$ confidence regions, respectively. The cosmological parameters with the largest likelihood are $\Omega_{m}=0.24_{-0.14}^{+0.16}$ and $w_{0}=-0.85_{-0.51}^{+0.28}(1 \sigma)$ with $\chi_{\min }^{2}=727.32$ for 659 degrees of freedom. For a prior of $\Omega_{m}=$ 0.29 , we obtain $w_{0}=-0.95_{-0.18}^{+0.14}$, which is consistent with the cosmological constant (i.e., $w_{0}=-1$ ) in a $1 \sigma$ confidence region.

3.4. $w(z)=w_{0}+w_{1} z /(1+z)$ Model: Time-Dependent Equation of State. We next examine models in which dark energy changes with time. As shown above, we adopt a simple model in which the dark energy equation of state can be parameterized by $[4,5]$

$$
w(z)=w_{0}+\frac{w_{1} z}{(1+z)}
$$

The $\Lambda$ CDM model is recovered when $w_{0}=-1$ and $w_{1}=0$. In this dark energy model, the luminosity distance is calculated by

$$
\begin{aligned}
D_{L}(z)= & c H_{0}^{-1}(1+z) \\
& \quad \cdot \int_{0}^{z} d z\left[(1+z)^{3} \Omega_{m}+\left(1-\Omega_{m}\right)\right. \\
& \left.\cdot(1+z)^{3\left(1+w_{0}+w_{1}\right)} e^{-3 w_{1} z /(1+z)}\right]^{-1 / 2} .
\end{aligned}
$$

Figure 6 shows the constraints on $w_{0}$ versus $w_{1}$ from $1 \sigma$ to $3 \sigma$ confidence regions. The dark cyan dash-dotted lines and blue dashed lines represent the constraints from 82 GRBs and $580 \mathrm{SNe}$ Ia, respectively. The red solid contours are obtained from the combination of these data. For a prior of $\Omega_{m}=0.29$,

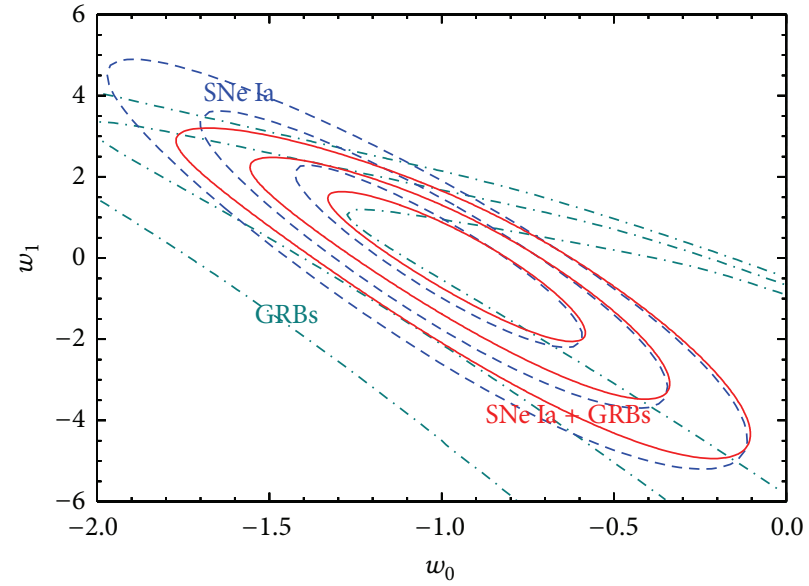

Figure 6: Constraints on $w_{0}$ and $w_{1}$ from $1 \sigma$ to $3 \sigma$ confidence regions with dark energy whose equation state is $w(z)=w_{0}+$ $w_{1} z /(1+z)$. The contours are derived from GRBs (dark cyan dashdotted lines), SNe Ia (blue dashed lines), and SNe Ia + GRBs (red solid lines), respectively.

we find the best dark energy parameters set is $\left(w_{0}, w_{1}\right)=$ $\left(-0.96_{-0.36}^{+0.39},-0.04_{-1.96}^{+1.72}\right)$ at the $1 \sigma$ confidence level with $\chi_{\min }^{2}=$ $727.54 / 659$. This result is also consistent with the $\Lambda \mathrm{CDM}$ model (i.e., $w_{0}=-1$ and $w_{1}=0$ ) in the $1 \sigma$ confidence region.

\section{Conclusions and Discussion}

In this paper, we have updated five GRB luminosity relations $\left(\tau_{\mathrm{lag}}-L, E_{p}-E_{\mathrm{iso}}, E_{p}-L, E_{p}-E_{\gamma}\right.$, and $\left.\tau_{\mathrm{RT}}-L\right)$ among certain spectral and light-curve features with the latest 139 GRBs. We find that the five relations indeed exist with the latest GRBs data. To avoid any assumption on cosmological models, we obtained the distance moduli of 57 low- $z(z<1.4)$ GRBs by using cubic spline interpolation from the 580 Union 2.1 SNe Ia compiled in [36]. Then, we calibrated the five relations with these 57 low- $z$ GRBs. In order to constrain cosmological models, we extended the five calibrated luminosity relations to high $-z$ and derived the distance moduli of 82 high $-z(z>$ 1.4) GRBs.

Motivated by these significant updates of the observational data, we considered the joint constraints on the Cardassian expansion model and dark energy with 580 Union2.1 SNe Ia sample $(z<1.4)$ and 82 calibrated GRBs' data $(1.4<z \leq 8.2)$. In the $\Lambda \mathrm{CDM}$ cosmology, we find that adding 82 high- $z$ GRBs to 580 SNe Ia significantly improves the constraint on $\Omega_{m}-\Omega_{\Lambda}$ plane. We obtain $\Omega_{m}=0.27_{-0.06}^{+0.08}$ and $\Omega_{\Lambda}=0.62_{-0.19}^{+0.18}(1 \sigma)$. For a flat universe, the contours yield $\left(\Omega_{m}, \Omega_{\Lambda}\right)=\left(0.29_{-0.04}^{+0.04}, 0.71_{-0.04}^{+0.04}\right)$. The transition redshift at which the universe switched from deceleration to acceleration phase is $z_{T}=0.64_{-0.14}^{+0.08}(1 \sigma)$. In the Cardassian expansion model, we obtain $\Omega_{m}=0.24_{-0.15}^{+0.15}$ and $n=0.16_{-0.52}^{+0.30}(1 \sigma)$. This result is consistent with the $\Lambda \mathrm{CDM}$ cosmology $(n=0)$ in the $1 \sigma$ confidence region, in agreement with the conclusions of [40]. We also fit two dark energy models, including the flat constant $w$ model (i.e., 
$w(z)=w_{0}$ ) and the time-dependent $w$ model (i.e., $w(z)=$ $\left.w_{0}+w_{1} z /(1+z)\right)$. Based on our analysis, it can be seen that our universe at higher redshift up to $z=8.2$ is consistent with the concordance model $\left(\Omega_{m}=0.27, \Omega_{\Lambda}=0.73, w_{0}=-1\right.$, and $\left.w_{1}=0\right)$ within $1 \sigma$ level. These results suggest that time dependence of the dark energy is small even if it exists.

Since the discoveries of distance indicators of GRBs, these luminosity indicators have been used as standard candles for cosmological research at high redshifts. However, the dispersion of distance indicators is still large, which restricted the precision of distance measurement by GRBs. The large dispersion may be due to the fact that some contamination of the GRB sample is unavoidable and that pure luminosity indicators may never be found for these sources. Of course, it could also be due to the fact that we simply have not yet identified the correct spectral and light-curve features to use for these luminosity relations. On the other hand, it could also be due to the fact that we are inevitably suffering from the systematic errors and intrinsic scatter associated with the data. In order to estimate distance of GRBs more precisely, we should take efforts to investigate possible origins of dispersion of the distance indicators and/or search for more precise distance indicators in the future.

\section{Conflict of Interests}

The authors declare that there is no conflict of interests regarding the publication of this paper.

\section{Acknowledgments}

This work is partially supported by the National Basic Research Program ("973" Program) of China (Grants 2014CB845800 and 2013CB834900), the National Natural Science Foundation of China (Grants nos. 11322328 and 11373068), the One-Hundred-Talents Program, the Youth Innovation Promotion Association, and the Strategic Priority Research Program "The Emergence of Cosmological Structures" (Grant no. XDB09000000) of the Chinese Academy of Sciences, and the Natural Science Foundation of Jiangsu Province (grant no. BK2012890).

\section{References}

[1] S. Perlmutter, G. Aldering, M. Della Valle et al., "Discovery of a supernova explosion at half the age of the Universe," Nature, vol. 391, no. 6662, pp. 51-54, 1998.

[2] B. P. Schmidt, N. B. Suntzeff, M. M. Phillips et al., "The high-Z supernova search: measuring cosmic deceleration and global curvature of the universe using type IA supernovae," The Astrophysical Journal, vol. 507, no. 1, pp. 46-63, 1998.

[3] A. G. Riess, A. V. Filippenko, P. Challis et al., "Observational evidence from supernovae for an accelerating universe and a cosmological constant," The Astronomical Journal, vol. 116, no. 3, pp. 1009-1038, 1998.

[4] M. Chevallier and D. Polarski, "Accelerating universes with scaling dark matter," International Journal of Modern Physics D, vol. 10, no. 2, pp. 213-223, 2001.
[5] E. V. Linder, "Exploring the expansion history of the universe," Physical Review Letters, vol. 90, no. 9, Article ID 091301, 2003.

[6] K. Freese and M. Lewis, "Cardassian expansion: a model in which the universe is flat, matter dominated, and accelerating," Physics Letters, Section B: Nuclear, Elementary Particle and HighEnergy Physics, vol. 540, no. 1-2, pp. 1-8, 2002.

[7] D. O. Jones, S. A. Rodney, A. G. Riess et al., "The discovery of the most distant known type Ia supernova at redshift 1.914," The Astrophysical Journal, vol. 768, no. 2, article 166, 2013.

[8] M. J. Sholl, M. L. Lampton, G. Aldering et al., "SNAP telescope," Optical, Infrared, and Millimeter Space Telescopes, vol. 5487, pp. 1473-1483, 2004.

[9] D. Q. Lamb and D. E. Reichart, "Gamma-ray bursts as a probe of the very high redshift universe," The Astrophysical Journal, vol. 536, no. 1, pp. 1-18, 2000.

[10] N. R. Tanvir, D. B. Fox, A. J. Levan et al., "A $\gamma$-ray burst at a redshift of $z \approx 8.2$," Nature, vol. 461, no. 7268, pp. 1254-1257, 2009.

[11] A. Cucchiara, A. J. Levan, D. B. Fox et al., "A photometric redshift of $z \sim 9.4$ for GRB 090429B," The Astrophysical Journal, vol. 736, article 7, 2011.

[12] J. P. Norris, G. F. Marani, and J. T. Bonnell, “Connection between energy-dependent lags and peak luminosity in gamma-ray bursts," The Astrophysical Journal, vol. 534, no. 1, pp. 248-257, 2000.

[13] E. E. Fenimore and E. Ramirez-Ruiz, "Redshifts for 220 BATSE gamma-ray bursts determined by variability and the cosmological consequences," http://arxiv.org/abs/astro-ph/0004176.

[14] L. Amati, F. Frontera, M. Tavani et al., "Intrinsic spectra and energetics of BeppoSAX gamma-ray bursts with known redshifts," Astronomy \& Astrophysics, vol. 390, no. 1, pp. 81-89, 2002.

[15] D. M. Wei and W. H. Gao, "Are there cosmological evolution trends on gamma-ray burst features?" Monthly Notices of the Royal Astronomical Society, vol. 345, no. 3, pp. 743-746, 2003.

[16] D. Yonetoku, T. Murakami, T. Nakamura, R. Yamazaki, A. K. Inoue, and $\mathrm{K}$. Ioka, "Gamma-ray burst formation rate inferred from the spectral peak energy-peak luminosity relation," The Astrophysical Journal, vol. 609, no. 2, pp. 935-951, 2004.

[17] G. Ghirlanda, G. Ghisellini, and D. Lazzati, "The collimationcorrected gamma-ray burst energies correlate with the peak energy of their $v F_{v}$ spectrum," The Astrophysical Journal, vol. 616, no. 1, pp. 331-338, 2004.

[18] B. E. Schaefer, "The hubble diagram to redshift $>6$ from 69 gamma-ray bursts," The Astrophysical Journal, vol. 660, no. 1, pp. 16-46, 2007.

[19] F.-Y. Wang, S. Qi, and Z.-G. Dai, “The updated luminosity correlations of gamma-ray bursts and cosmological implications," Monthly Notices of the Royal Astronomical Society, vol. 415, no. 4, pp. 3423-3433, 2011.

[20] B. E. Schaefer, "Gamma-ray burst Hubble diagram to $\mathrm{z}=4.5$," The Astrophysical Journal Letters, vol. 583, no. 2, pp. L67-L70, 2003.

[21] Z. G. Dai, E. W. Liang, and D. Xu, "Constraining $\Omega_{M}$ and dark energy with gamma-ray bursts," The Astrophysical Journal Letters, vol. 612, no. 2, pp. L101-L104, 2004.

[22] E. Liang and B. Zhang, "Model-independent multivariable gamma-ray burst luminosity indicator and its possible cosmological implications," The Astrophysical Journal, vol. 633, no. 2, pp. 611-623, 2005. 
[23] Y. Kodama, D. Yonetoku, T. Murakami, S. Tanabe, R. Tsutsui, and T. Nakamura, "Gamma-ray bursts in $1.8<z<5.6$ suggest that the time variation of the dark energy is small," Monthly Notices of the Royal Astronomical Society, vol. 391, no. 1, pp. L1L4, 2008 .

[24] R. Tsutsui, T. Nakamura, D. Yonetoku et al., "Constraints on $\omega_{0}$ and $\omega_{a}$ of dark energy from high-redshift gamma-ray bursts," Monthly Notices of the Royal Astronomical Society: Letters, vol. 394, no. 1, pp. L31-L35, 2009.

[25] J.-J. Wei, X.-F. Wu, and F. Melia, "The gamma-ray burst hubble diagram and its implications for cosmology," The Astrophysical Journal, vol. 772, no. 1, article 43, 2013.

[26] G. Ghirlanda, G. Ghisellini, D. Lazzati, and C. Firmani, "Gamma-ray bursts: new rulers to measure the universe," The Astrophysical Journal Letters, vol. 613, no. 1, pp. L13-L16, 2004.

[27] G. Ghirlanda, G. Ghisellini, C. Firmani, L. Nava, F. Tavecchio, and D. Lazzati, "Cosmological constraints with GRBs: homogeneous medium vs. wind density profile," Astronomy and Astrophysics, vol. 452, no. 3, pp. 839-844, 2006.

[28] E. Liang and B. Zhang, "Calibration of gamma-ray burst luminosity indicators," Monthly Notices of the Royal Astronomical Society, vol. 369, no. 1, pp. L37-L41, 2006.

[29] C. Firmani, G. Ghisellini, G. Ghirlanda, and V. Avila-Reese, "A new method optimized to use gamma-ray bursts as cosmic rulers," Monthly Notices of the Royal Astronomical Society: Letters, vol. 360, no. 1, pp. L1-L15, 2005.

[30] F. Y. Wang and Z. G. Dai, "Constraining the cosmological parameters and transition redshift with gamma-ray bursts and supernovae," Monthly Notices of the Royal Astronomical Society, vol. 368, no. 1, pp. 371-378, 2006.

[31] L. Amati, C. Guidorzi, F. Frontera et al., "Measuring the cosmological parameters with the $E_{p, i}-E_{i s o}$ correlation of gamma-ray bursts," Monthly Notices of the Royal Astronomical Society, vol. 391, no. 2, pp. 577-584, 2008.

[32] H. Li, M. Su, Z. Fan, Z. Dai, and X. Zhang, "Constraints on dark energy models including gamma ray bursts," Physics Letters B, vol. 658, no. 4, pp. 95-100, 2008.

[33] N. Liang, W. K. Xiao, Y. Liu, and S. N. Zhang, "A cosmologyindependent calibration of gamma-ray burst luminosity relations and the hubble diagram," The Astrophysical Journal, vol. 685, no. 1, pp. 354-360, 2008.

[34] H. Wei and S.-N. Zhang, "Reconstructing the cosmic expansion history up to redshift $z=6.29$ with the calibrated gamma-ray bursts," The European Physical Journal C, vol. 63, no. 1, pp. 139$147,2009$.

[35] H. Wei, "Observational constraints on cosmological models with the updated long gamma-ray bursts," Journal of Cosmology and Astroparticle Physics, vol. 2010, no. 8, article 20, 2010.

[36] N. Suzuki, D. Rubin, C. Lidman et al., "The hubble space telescope cluster supernova survey. V. Improving the darkenergy constraints above $\mathrm{z}>1$ and building an early-typehosted supernova sample," The Astrophysical Journal, vol. 746, no. 1, article 85, 2012.

[37] D. Yonetoku, T. Murakami, R. Tsutsui, T. Nakamura, Y. Morihara, and K. Takahashi, "Possible origins of dispersion of the peak energy-brightness correlations of gamma-ray bursts," Publications of the Astronomical Society of Japan, vol. 62, no. 6, pp. 1495-1507, 2010.

[38] G. Ghirlanda, G. Ghisellini, C. Firmani, A. Celotti, and Z. Bosnjak, "The peak luminosity-peak energy correlation in gamma-ray bursts," Monthly Notices of the Royal Astronomical Society, vol. 360, no. 1, pp. L45-L49, 2005.
[39] A. G. Riess, L.-G. Sirolger, J. Tonry et al., “Type Ia supernova discoveries at $z>1$ from the Hubble Space Telescope: evidence for past deceleration and constraints on dark energy evolution," The Astrophysical Journal, vol. 607, no. 2, pp. 665-687, 2004.

[40] C.-J. Feng and X.-Z. Li, "Cardassian universe constrained by latest observations," Physics Letters B, vol. 692, no. 2, pp. 152$156,2010$. 

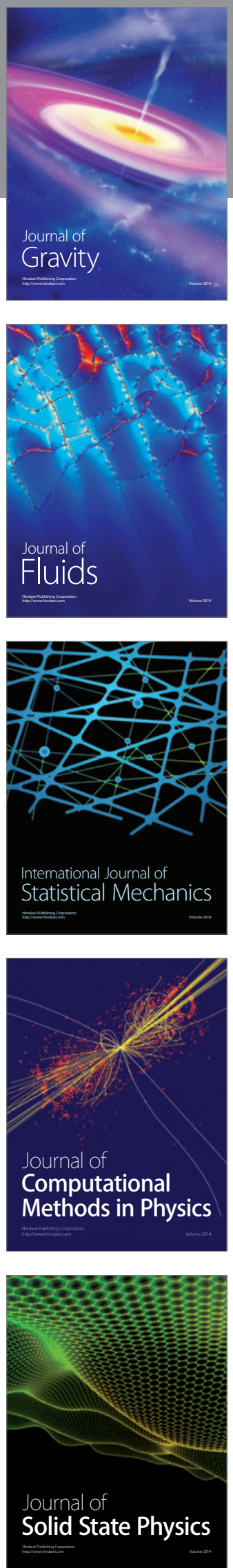

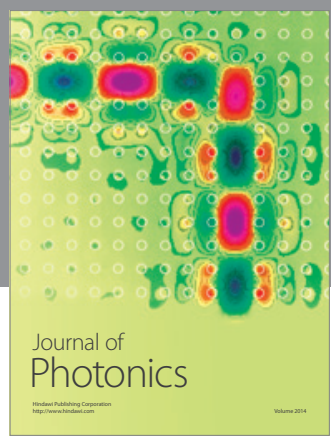

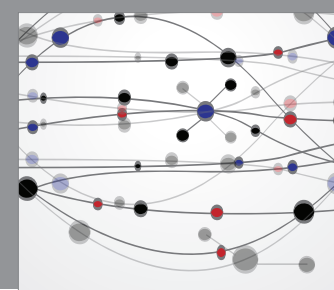

The Scientific World Journal

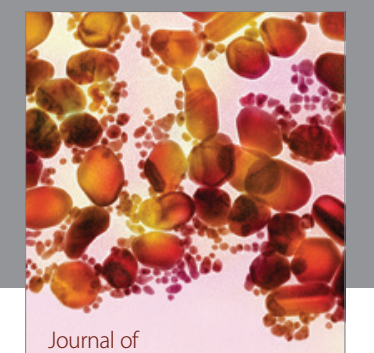

Soft Matter
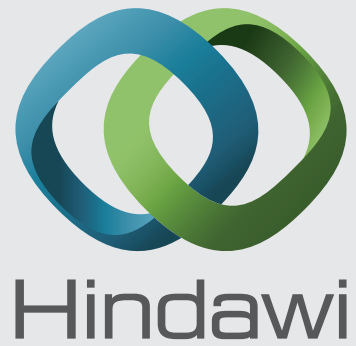

Submit your manuscripts at

http://www.hindawi.com
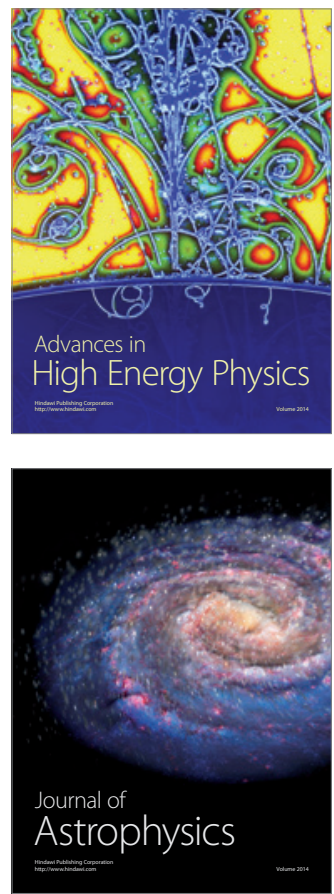
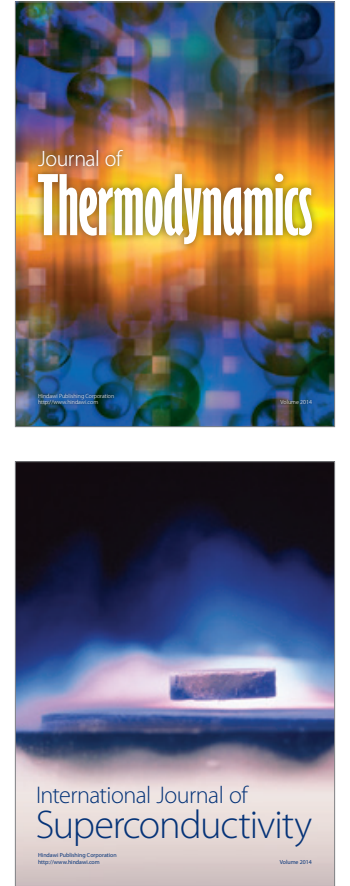
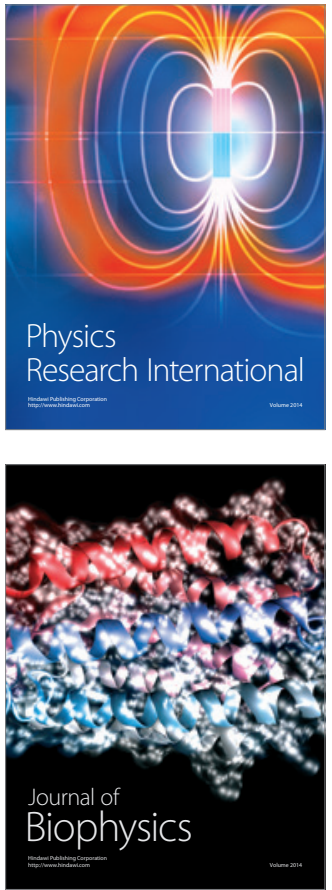
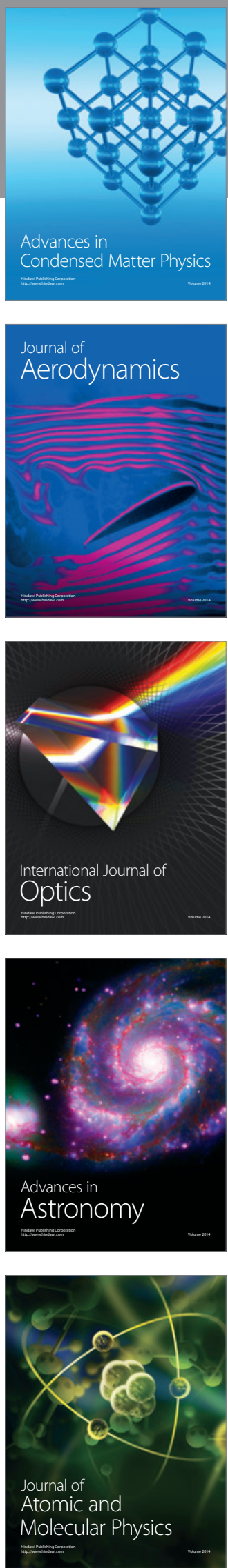